Cite this: Phys. Chem. Chem. Phys. 2014, 16, 9259

Received 7th February 2014 Accepted 17th March 2014

DOI: 10.1039/c4cp00567h

www.rsc.org/pccp

\title{
A comparative structural study in monolayers of GPI fragments and their binary mixtures $\dagger$
}

\author{
C. Stefaniu, ${ }^{\star a}$ I. Vilotijevic, ${ }^{a}$ G. Brezesinski, ${ }^{a}$ P. H. Seeberger ${ }^{a b}$ and D. Varón Silva ${ }^{\star a b}$
}

Glycosylphosphatidylinositols (GPIs), natural complex glycolipids essential for a range of biological functions, are poorly understood with regard to their interactions and arrangements in cellular membranes. To evaluate the role of the head group in the structure formation in 2D model membranes (monolayers formed at the soft air/liquid interface), we employed the highly surface sensitive grazing incidence $X$-ray diffraction technique to investigate three GPI-fragments bearing the same hydrophobic part but different head groups. Condensed monolayers of simple GPI fragments are defined only by ordered alkyl chains. The monolayers of more complex fragments are additionally characterized by highly ordered head groups. Due to the strong $\mathrm{H}$-bond network formed by the head groups, GPI-fragment 3 both segregates and induces order into a model membrane phospholipid (POPC) that mimics the liquiddisordered phase of cell membranes. Here, we show that the strong van der Waals interactions between hydrophobic chains overcome the head group interactions and dominate the structure formation in mixtures of GPI-fragment 3 with lipids that form liquid-condensed phases. This behaviour can be linked to the GPIs affinity for the lipid rafts.

\section{Introduction}

Glycosylphosphatidylinositols (GPIs) are natural complex glycolipids present on the outer leaflet of eukaryotic cell membranes. ${ }^{1-3}$ Existing as free GPIs or as part of GPI-anchored proteins (GPI-APs), they are involved in a range of vital biological functions such as signal transduction, intermembrane transport, protein sorting and trafficking. ${ }^{4-8}$ Their affinity for temporarily existent liquidordered membrane microdomains (lipid rafts), although not well understood, is postulated to play a role in important biological processes. ${ }^{9-12}$ Details about the structural arrangement of GPIs in cellular membranes could provide a better understanding of the chemical structure/structural membrane arrangement/biological function relationship for GPI glycolipids and the contributions of GPIs to the function of the attached protein that could have wide biomedical implications.

To establish a correlation between the chemical structure of the glycan moiety of GPI glycolipids and their structural arrangement in 2D model membranes, we conducted a systematic comparative study of GPI-fragment monolayers starting with a typical lipid of a mature GPI, ${ }^{13}$ diacylglycerol $\mathbf{1}$, and expanding towards the

\footnotetext{
${ }^{a}$ Max Planck Institute of Colloids and Interfaces, 14424 Potsdam, Germany. E-mail: stefaniu@mpikg.mpg.de, daniel.varon@mpikg.mpg.de

${ }^{b}$ Department of Chemistry and Biochemistry, Free University of Berlin, Arnimallee 22, 14195 Berlin, Germany

$\dagger$ Electronic supplementary information (ESI) available: Detailed experimental procedures including GIXD results and analysis. See DOI: 10.1039/c4cp00567h
}

more complex structures including phosphatidyloinositol 2 and zwitterionic GPI glycolipid 3 (Fig. 1) ${ }^{14,15}$ With respect to the affinity of GPIs for lipid rafts, we have previously demonstrated that compound 3 both segregates and induces order in mixed monolayers with POPC (mimic of the liquid disordered phase of cell membranes). ${ }^{16}$ To further the understanding of these effects, we have investigated the ability of compound 3 to mix with lipids that form liquid-condensed phases and evaluated miscibility behaviour of compound $\mathbf{3}$ with compounds $\mathbf{1}$ or $\mathbf{2}$ in two-component monolayers. Here, we report the results of these studies that

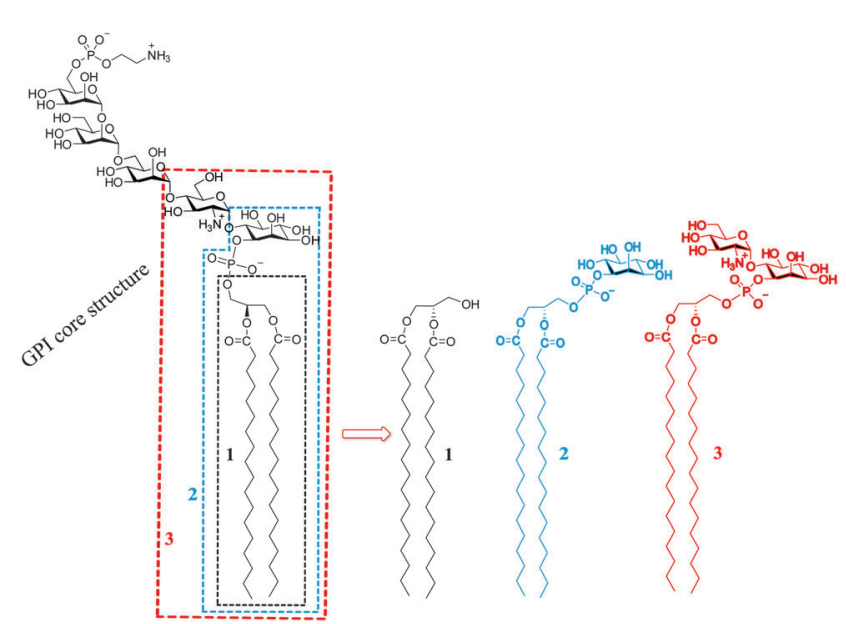

Fig. 1 Structures of the conserved GPI core and of the three GPI-fragments investigated in this study. 
provide a description of the molecular ordering in investigated model membranes with angstrom-level resolution and offer insights into interactions between structurally different lipids.

\section{Results and discussion}

\section{Single component monolayers}

To obtain information on phase transitions in 2D model membranes of 1,2-distearoyl-sn-glycerol (1), Langmuir isotherms were measured on an aqueous subphase at $20{ }^{\circ} \mathrm{C}$ (Fig. 2B). The isotherm suggests that the monolayer of $\mathbf{1}$ is fully condensed at all surface pressures above zero and exhibits a first-order phase transition from a gaseous (G) to a liquid-condensed (LC) phase at close to zero lateral pressure. The change in slope at $12.8 \mathrm{mN} \mathrm{m}^{-1}$ (Fig. 2B) indicates a second-order phase transition leading to an almost incompressible monolayer. The monolayer structural parameters have been determined by GIXD measurements. The selected contour plots obtained at different surface pressures are represented (Fig. 2A) as a function of the in-plane scattering vector component $Q_{x y}$ and the out-of-plane scattering vector component $Q_{z}$. Only for the lowest surface pressure $\left(3.3 \mathrm{mN} \mathrm{m}^{-1}-\right.$ Fig. $2 \mathrm{~A}$ and Tables, ESI $\dagger$ ) the monolayer structure of $\mathbf{1}$ is represented by three diffraction peaks. Such an intensity distribution is characteristic for an oblique (chiral) chain lattice. ${ }^{17,18}$ The maxima of the Bragg rods are above the horizon, indicating tilted alkyl chains with respect to the surface normal. The influence of the chirality in glycerol on the lattice structure is diminished by strong van der Waals interactions between the two $\mathrm{C}_{18}$ alkyl chains of $\mathbf{1}$ at higher compression states. The monolayer structure changes above $5 \mathrm{mN} \mathrm{m}^{-1}$ into a distorted hexagonal phase (the tilted acyl chains pointing towards the nearest neighbour - $\mathrm{NN}, \mathrm{L}_{2}$ phase), and above $15 \mathrm{mN} \mathrm{m}^{-1}$ into a hexagonal unit cell of non-tilted chains (LS phase).
For all surface pressures the Bragg rods are characterized by a full-width at half-maximum (FWHM) of $0.255 \AA^{-1}$. This value is directly correlated with the length of the scattering unit $(L)$ by the relationship: $\operatorname{FWHM}\left(Q_{z}\right)=0.9 \times(2 \pi) / L$. The value of $21.7 \AA$ is in good agreement with the length of the extended $\mathrm{C}_{18}$ chain assuming all-trans conformation ${ }^{19,20}$ and confirms the existence of a monolayer. Two additional parameters have been determined by extrapolation of the GIXD data (Tables, ESI $\dagger$ ). The transition surface pressure of $13 \mathrm{mN} \mathrm{m}^{-1}$, the lateral pressure at which the acyl chains become untilted with respect to the normal, was determined by extrapolation of $1 / \cos (t)$ to a zero tilt angle (Fig. 2C). This value is in good agreement with the data derived from the isotherm (Fig. 2B, inflection point at $12.8 \mathrm{mN} \mathrm{m}^{-1}$ ). The maximum tilt angle of the acyl chains was determined by extrapolation to zero surface pressure and it amounts to $19^{\circ}$ for $\mathbf{1}$ (Fig. 2C). Comparison of the surface pressure-molecular area dependencies acquired from the Langmuir balance and GIXD reveals a shift towards smaller areas in the compression isotherms obtained from the diffraction experiments. This shift can be explained by the fact that GIXD detects only the well-ordered regions while the Langmuir isotherm measures an averaged area values including empty spaces (voids) in the condensed Langmuir layers. ${ }^{21} \mathrm{~A}$ linear dependence of the lattice distortion $d v s \cdot \sin ^{2}(t)$ is in agreement with a modified Landau theory. ${ }^{22}$ The measured zero distortion at zero tilt indicates that the lattice distortion at lower pressures is caused only by tilting of the molecules (Fig. 2D).

The more complex GPI-fragment 2 (Fig. 1 and 3) is characterized by a bulkier head group featuring a phosphoinositol that can be ionized depending on the acid-base equilibrium in the surrounding medium. Surface pressure/molecular area $(\pi / A)$ isotherms for 2 have been recorded at the air/water interface at $20{ }^{\circ} \mathrm{C}$. In addition to the measurements on the pure water subphase, measurements were also carried out on an aqueous subphase containing $\mathrm{Ca}^{2+}$ ions $\left(2 \mathrm{mM} \mathrm{CaCl}_{2}\right)$ that could provide insight

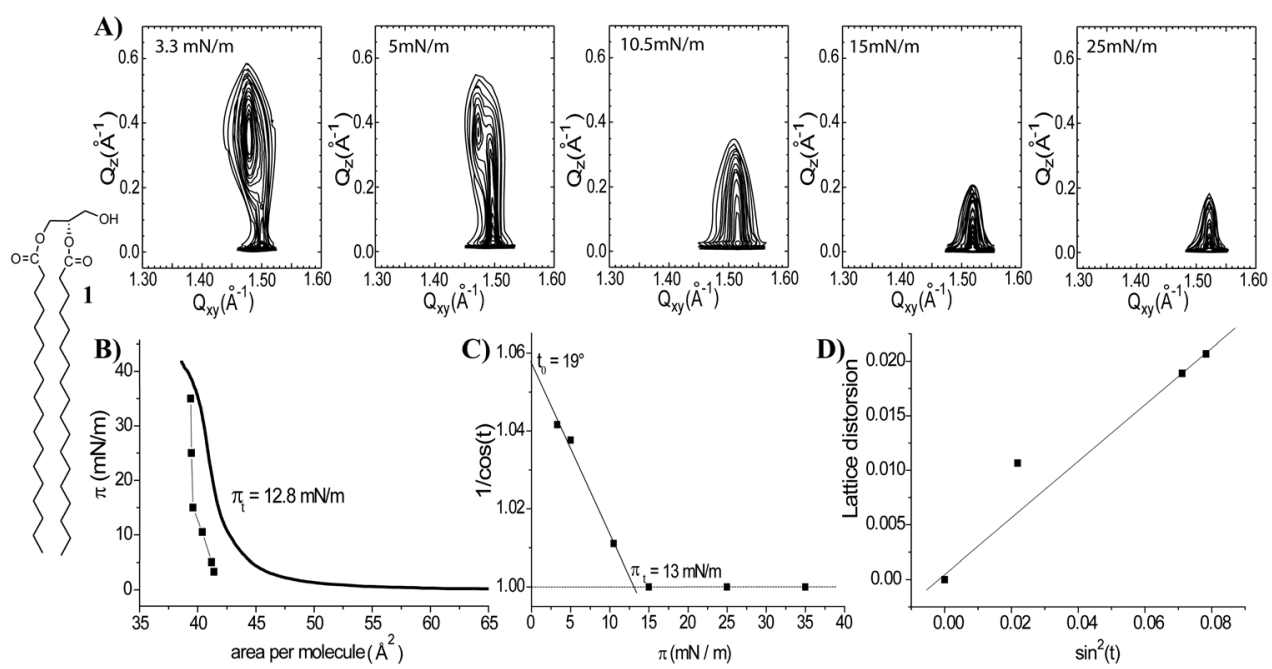

Fig. 2 (A) GIXD patterns of monolayers of 1 (measured on water at $20^{\circ} \mathrm{C}$ ) at different lateral pressures (indicated); (B) comparison between surface pressure-molecular area isotherms determined by GIXD (square data points) and with the Langmuir balance (continuous line); (C) variation of the tilt angle of the alkyl chains $(t)$ represented as $1 / \cos (t)$ with the lateral surface pressure $(\pi)$. Extrapolation of $1 / \cos (t)$ to 1 gives the transition surface pressure $\left(\pi_{t}\right)$ to an untilted state, while the extrapolation to $\pi=0$ yields the maximum tilt angle $\left(t_{0}\right)$ of the chains; (D) lattice distortion versus $\sin ^{2}(t)$. 

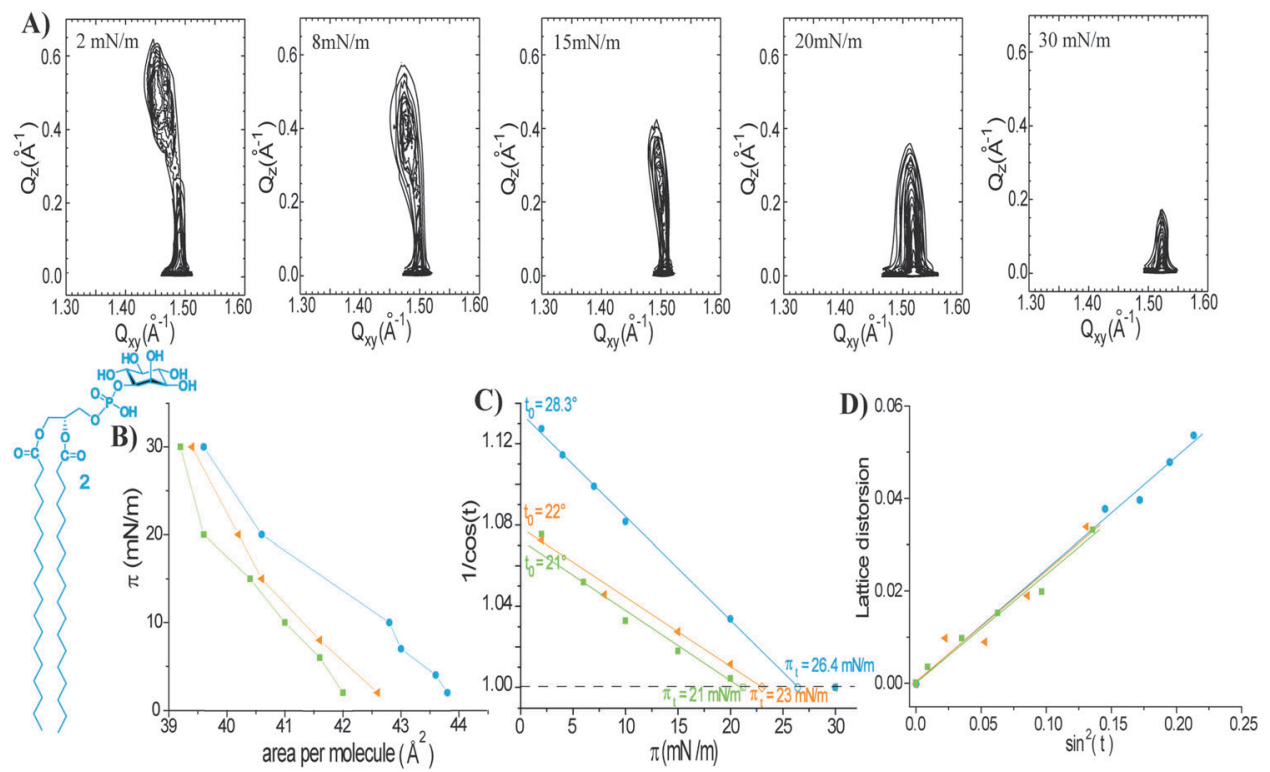

Fig. 3 (A) GIXD patterns of monolayers of 2 (measured on water at $20^{\circ} \mathrm{C}$ ) depicting the change in the monolayer structure upon lateral compression; (B) surface pressure-molecular area isotherms determined by GIXD on different subphases: $2 \mathrm{mM} \mathrm{CaCl} 2$ aqueous solution - green squares; pure water orange triangles; and PBS $(10 \mathrm{mM}, \mathrm{pH} 7.4,150 \mathrm{mM} \mathrm{NaCl})$ - blue circles $(\mathrm{C})$ variation of the tilt angle of the alkyl chains $(t)$ represented as $1 / \mathrm{cos}(t)$ with the lateral surface pressure $(\pi)$ at $20^{\circ} \mathrm{C}$ on different subphases: $2 \mathrm{mM} \mathrm{CaCl}_{2}$ aqueous solution - green squares; pure water - orange triangles; and PBS - blue circles. Extrapolation of $1 / \cos (t)$ to 1 gives the transition surface pressure $\left(\pi_{t}\right)$ at which the tilt angle becomes zero, while the extrapolation to $\pi=0$ yields the maximum tilt angle $\left(t_{0}\right)$ of the chains; $(D)$ lattice distortion versus $\sin ^{2}(t)$ on different subphases: $2 \mathrm{mM} \mathrm{CaCl} 2$ aqueous solution - green squares; pure water - orange triangles; and PBS - blue circles.

into the interactions of $\mathrm{Ca}^{2+}$ ions with the anionic phosphate groups and the possible formation of calcium phosphate bridges between molecules of 2 . The phosphate buffer (PBS) (10 mM, pH 7.4, $150 \mathrm{mM} \mathrm{NaCl}$ ) subphase was used to investigate the changes in the intermolecular interactions that may arise from different degrees of phosphate ionization. All recorded isotherms suggest a phase transition from a gaseous to a LC phase (data not shown).

For depicting the changes of the monolayer structure with increased surface pressure, a few representative contour plots for monolayers of 2 are shown in Fig. 3A. The subphase composition has a clear influence on the packing properties (Fig. 3B and C). The in-plane area slightly increases going from the $\mathrm{CaCl}_{2}$ subphase, to water and PBS (Fig. 3B). These variations are also reflected in the different linear plots of the chain tilt angle $(1 / \cos (t))$ versus the lateral pressure. In the presence of $\mathrm{Ca}^{2+}$ ions (Fig. 3B, green squares), the formation of calcium phosphate bridges between molecules of 2 induces tighter packing. ${ }^{23}$ The GIXD data (Tables, $\mathrm{ESI}+$ ) reveal that on the $\mathrm{Ca}^{2+}$ containing subphase the monolayers of $\mathbf{2}$ are characterized by an oblique (chiral) chain lattice only below $5 \mathrm{mN} \mathrm{m}^{-1}$. Upon lateral compression, this lattice transforms into a distorted hexagonal phase, and finally, above $21 \mathrm{mN} \mathrm{m}^{-1}$, into a hexagonal one of non-tilted chains. For the monolayers of 2 prepared on the surface of pure water, the GIXD results are similar, yet slightly shifted to higher tilt angles because of the less tight packing (larger molecular area).

As shown in Fig. 3C (light blue circles and Tables, ESI $\dagger$ ), the higher ionization degree of the phosphate group on PBS (higher pH and higher ionic strength) compared to that on water leads to an increased effective area of the head groups by stronger electrostatic repulsions, inducing thus a much larger tilt angle of the hydrophobic chains. The monolayers of 2 prepared on PBS are characterized by an oblique (chiral) chain lattice, which transforms into a distorted hexagonal phase upon compression (slightly below $20 \mathrm{mN} \mathrm{m}^{-1}$ ). Only above $26.4 \mathrm{mN} \mathrm{m}^{-1}$, the monolayer structure changes to hexagonal packing of untilted chains (LS phase). Plots of the lattice distortion $d$ versus $\sin ^{2}(t)$ for monolayers of 2 formed on the different subphases are identical (Fig. 3D). The correspondence of the zero tilt angle with zero distortion indicates that the lattice distortion in monolayers of 2 is caused only by tilting of the molecules as observed for $\mathbf{1}$.

The third and most complex compound of the series is the GlcN $\alpha 1 \rightarrow$ 6myoIno-1-phosphodistearylglycerol (3, Fig. 1 and 4) which in addition to the phosphoinositol (present in 2) carries a glucosamine unit. This fragment is considered to mimic the behaviour of GPIs because it features both the amino and phosphate groups responsible for the zwitterionic character of the charged GPI head groups. ${ }^{24}$ The GIXD data obtained with the GPI-fragment 3 proved to be complex. The plot features not only 3 Bragg reflections characteristic for ordered alkyl chains in an oblique lattice as described for compounds 1 and 2, but also additional 5 Bragg peaks. ${ }^{16}$ The GIXD data (Fig. 4A and B) for monolayers of $\mathbf{3}$ are consistent with the existence of two commensurate lattices: an oblique lattice of the alkyl chains characterized by a large tilt angle, and a molecular lattice based on strong interactions between highly ordered head groups (Fig. 4C). Compound 3 forms invariable monolayers with an enhanced order on subphases of different $\mathrm{pH}$ values $(2,6$, and 7.4$)$ and 


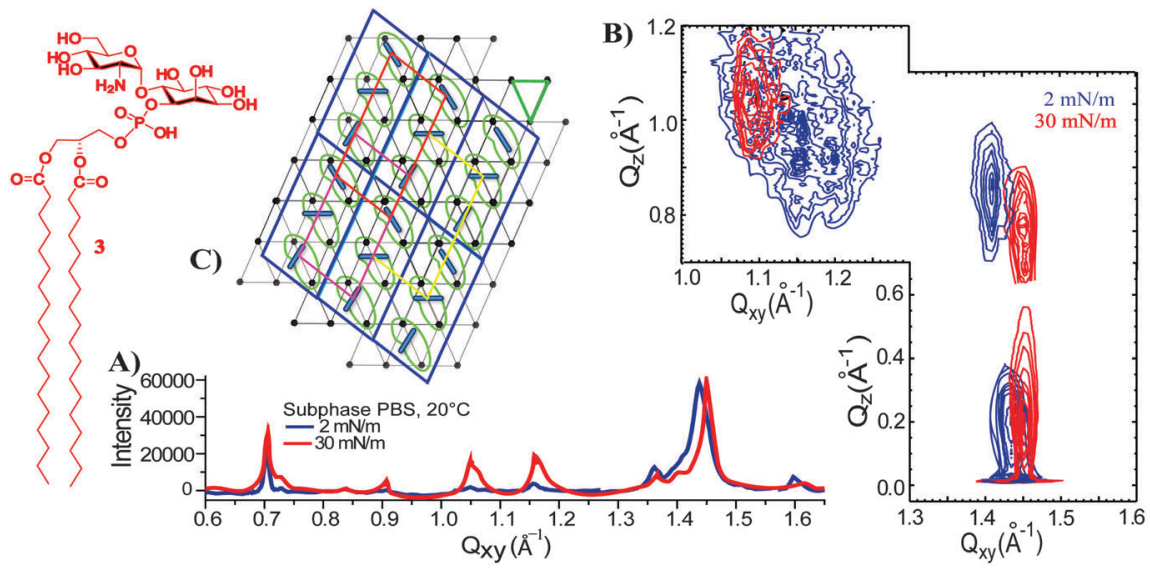

Fig. 4 (A) GIXD patterns and (B) GIXD contour plots of monolayers of 3 on PBS at $20{ }^{\circ} \mathrm{C}\left(2 \mathrm{mN} \mathrm{m}^{-1}-\right.$ blue line, and $30 \mathrm{mN} \mathrm{m}^{-1}-$ red line) depicting unusual slight changes in the monolayer structure upon lateral compression; (C) representation of the commensurable lattices describing the lateral order of the alkyl chains (black dots; repeating unit cell shown by the green triangle) and of the molecules (repeating unit cell shown by the blue parallelogram). Repeating unit cells (red, yellow, and magenta) link the molecules with the same head group orientation. Delimitation of the molecules in the lattice plane indicated by the green line: view from above.

ionic strengths (pure water, $2 \mathrm{mM} \mathrm{CaCl}_{2}$, or $150 \mathrm{mM} \mathrm{NaCl}$ ) suggesting that simple electrostatic interactions do not play a role in the ordering of molecules. This enhanced structural arrangement is a consequence of hydrogen bonding interactions between the head groups that lead to their rigid and highly ordered arrangement which controls the molecular packing. This agrees well with the lack of major changes in the monolayer structure upon lateral compression, and with the large tilt angle of the alkyl chains $\left(43^{\circ}\right)$. The hydrogen bonding network could be disrupted only on $5 \mathrm{M}$ urea subphases leading to the loss of the molecular lattice and the restructuring of the monolayer. ${ }^{16}$

\section{Comparative analysis of the monolayer structures of the investigated GPI-fragments}

Compound 1 features the smallest head group that allows a hexagonal arrangement of non-tilted chains above $13 \mathrm{mN} \mathrm{m}^{-1}$. The largest tilt angle is observed in the uncompressed state and amounts to $19^{\circ}$ corresponding to a molecular in-plane area of $41.9 \AA^{2}$ (see ESI $\dagger$ ). Only at low surface pressures, the monolayer structure is influenced by the chiral carbon atom in the head group (weak chiral interactions). ${ }^{25}$ Compound 2 bears additional phosphate and inositol moieties. In terms of the molecular shape concept ${ }^{26}$ one would expect a strong influence of the larger head group on the monolayer structure. However, the effective in-plane area $\left(42.7 \AA^{2}\right)$ of this head group on a water subphase is not substantially larger than the one of compound 1 showing that the additional moieties can be accommodated just beneath the glycerol. The phosphate group plays an important role, responding to changes in the subphase composition by changing its ionization degree and hydration shell. The bigger head group and the additional electrostatic repulsions induce larger tilt angles of the chains and a higher transition pressure $\left(26 \mathrm{mN} \mathrm{m}^{-1}\right)$ to a non-tilted phase. The molecular in-plane area in the uncompressed state amounts now to $44.8 \AA^{2}$. This value can be reduced by the action of divalent ions bridging neighbouring head groups.
This classical behaviour of compounds $\mathbf{1}$ and $\mathbf{2}$ can be explained by the mismatch of area requirements of the hydrophilic head groups and the hydrophobic chains. The crosssectional area of the chains amounts in all cases to $19.8 \AA^{2}$. The reduction of the effective in-plane area (orientation, hydration, interactions) of the head groups allows the chains to be untilted at higher packing densities at which the attractive van der Waals interactions between the chains overcome the steric and/ or electrostatic repulsive interactions between the head groups (Fig. 5).

The situation is completely different for compound 3 . The purely geometrical considerations fail to describe the unusual behaviour. The large tilt angle in the uncompressed state $\left(45.2^{\circ}\right)$ corresponds to a molecular area of $55.6 \AA^{2}$. In the light of the molecular shape concept, the large head group (conical shape) would lead to the formation of non-lamellar phases in bulk. In the present case, the molecules are confined to the planar interface and can optimize the attractive interactions between the chains only by strong tilting. The marginal change in the tilt angle during compression $\left(0.06^{\circ} /\left(\mathrm{mN} \mathrm{m}^{-1}\right)\right)$ indicates additional strong interactions (hydrogen bonds) between the head groups rigidifying the monolayer structure. Compound $\mathbf{1}$ with no additional attractive or repulsive interactions except the dispersion forces can be more easily compressed $\left(1.46 \%\left(\mathrm{mN} \mathrm{m}^{-1}\right)\right)$. The additional repulsive electrostatic interactions occurring between the head groups of compound 2 decrease this value to $\sim 1^{\circ} /\left(\mathrm{mN} \mathrm{m}^{-1}\right)$. The formation of the hydrogen bonding network between the zwitterionic GPI glycolipid head groups leads to a monolayer structure which basically cannot be changed by compression.

\section{Two-component monolayers}

The invariable nature of monolayers of 3 provoked questions about the potential role of head group interactions in the formation of clusters and rafts. This turned our attention to equimolar two-component monolayers containing $\mathbf{3}$ and POPC, a representative low-melting model membrane phospholipid 


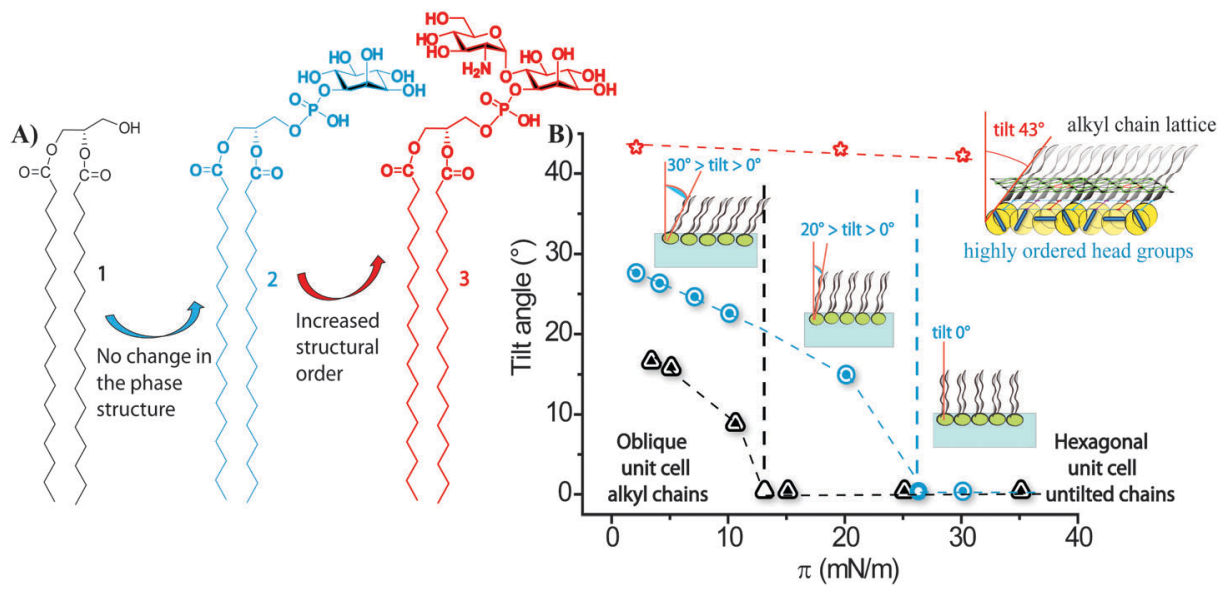

Fig. 5 (A) Chemical structures of the three investigated GPI-fragments (B) comparative representation of the monolayer structural changes of the three $\mathrm{GPI}$-fragments depicted by the variation of the tilt angle of the alkyl chains $(t)$ with the lateral surface pressure $(\pi)$ on PBS at $20{ }^{\circ} \mathrm{C}$.

which forms monolayers in a liquid-disordered state at $20{ }^{\circ} \mathrm{C} .{ }^{27}$ In this mixed two-component monolayer, a fraction of the GPI-fragment 3 is able to phase segregate due to strong head group interactions, while a certain percentage mixes with the liquid-disordered POPC ${ }^{16}$ inducing order in a highly cooperative way similar to sphingomyelin-POPC mixtures previously described as a representative model raft system..$^{28}$ Considering the broad implications of the interactions of GPI-APs and GPIs on processes in real cell membranes these effects may have far-reaching consequences. Further investigation is aimed to reveal whether the head group interactions of GPI-fragment 3 are strong enough to also induce clustering in condensed monolayers. To this end, we investigated mixed two-component monolayers of $\mathbf{3}$ with the GPI-fragments $\mathbf{1}$ and 2.

Two-component monolayers have been prepared by co-spreading mixtures of 1 and 3 (1:1 molar ratio) at the air/water interface. These monolayers are characterized by a homogeneous structure defined only by ordered alkyl chains (Fig. 6A). The absence of Bragg peaks at low $Q_{x y}$ values, characteristic of the supercell in the monolayers of GPI-fragment 3 (Fig. 4A), clearly indicates that the head group structure is not formed in these mixed monolayers.
The two components are miscible at a 1:1 molar ratio and the strong van der Waals interactions between the long alkyl chains do not allow the formation of the H-bond network between the head groups as formed in monolayers of pure compound 3.

In contrast to the extremely rigid monolayer structure of 3 (Fig. 4A), ${ }_{1}^{16}$ the orientation of the molecules in the binary mixture changes drastically with increasing surface pressure. The shift of the Bragg peaks to higher $Q_{x y}$ values and of the Bragg rods to lower $Q_{z}$ values (Fig. 6B and Tables, ESI $\dagger$ ) indicates a continuous change in the monolayer ordering from a distorted hexagonal phase (the tilted acyl chains pointing towards the nearest neighbour - NN, $\mathrm{L}_{2}$ phase) towards a hexagonal non-tilted phase (described by only one Bragg peak at high $Q_{x y}$ and zero $Q_{z}$ values, LS phase). The transition into the non-tilted state (Fig. S1, ESI $\dagger$ ) occurs at high surface pressures $\left(17 \mathrm{mN} \mathrm{m}^{-1}\right.$ and $20 \mathrm{mN} \mathrm{m}^{-1}$ for monolayers formed on the water and PBS subphase, respectively).

The addition of compound 1 to the monolayers of 3 not only disrupts the head group ordering but also changes the packing constraints of the molecules. The two alkyl chains of 1 and the small head group seem to compensate for the large head group in-plane area requirement of the GPI-fragment 3, causing tighter
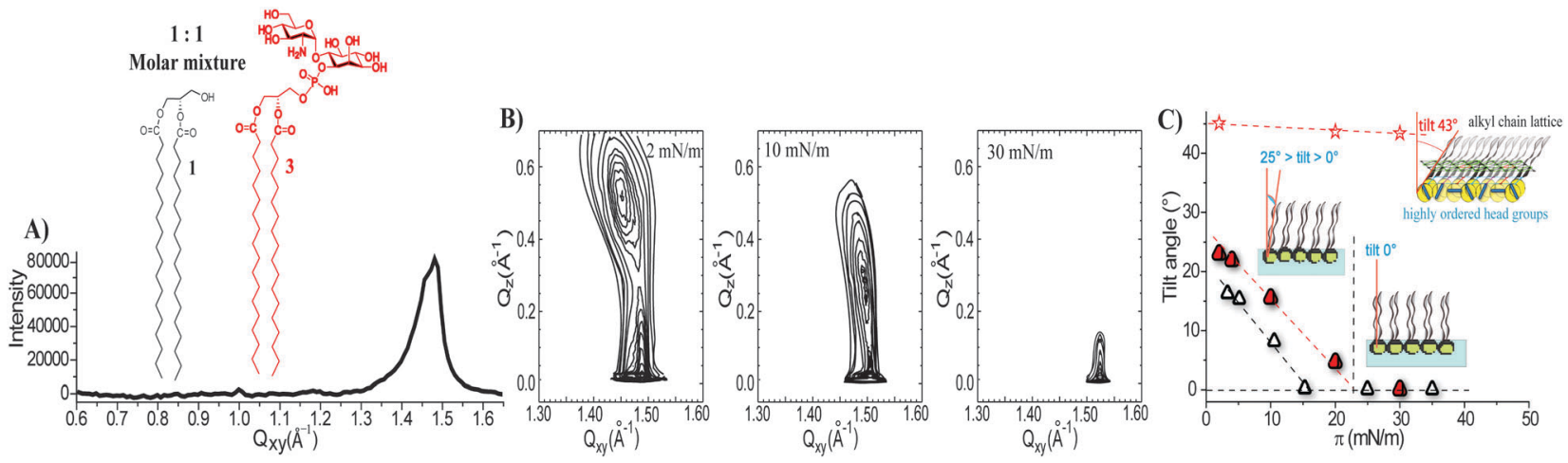

Fig. 6 (A) GIXD pattern of monolayers of the binary mixture of GPI-fragments 1 and $\mathbf{3}$ (molar ratio $1: 1$ ) prepared on PBS at $20{ }^{\circ} \mathrm{C}\left(2 \mathrm{mN} \mathrm{m}^{-1}\right.$ ); (B) GIXD contour plots of the same monolayers at different lateral surface pressures (indicated); (C) comparative representation of the monolayer structural changes of the pure GPI-fragments ( $\mathbf{1}$ - black empty triangles, $\mathbf{3}$ - red empty stars) and of their binary 1:1 molar mixture (1/3 - red filled triangles) depicted by the variation of the tilt angle of the alkyl chains $(t)$ with the lateral surface pressure $(\pi)$. 

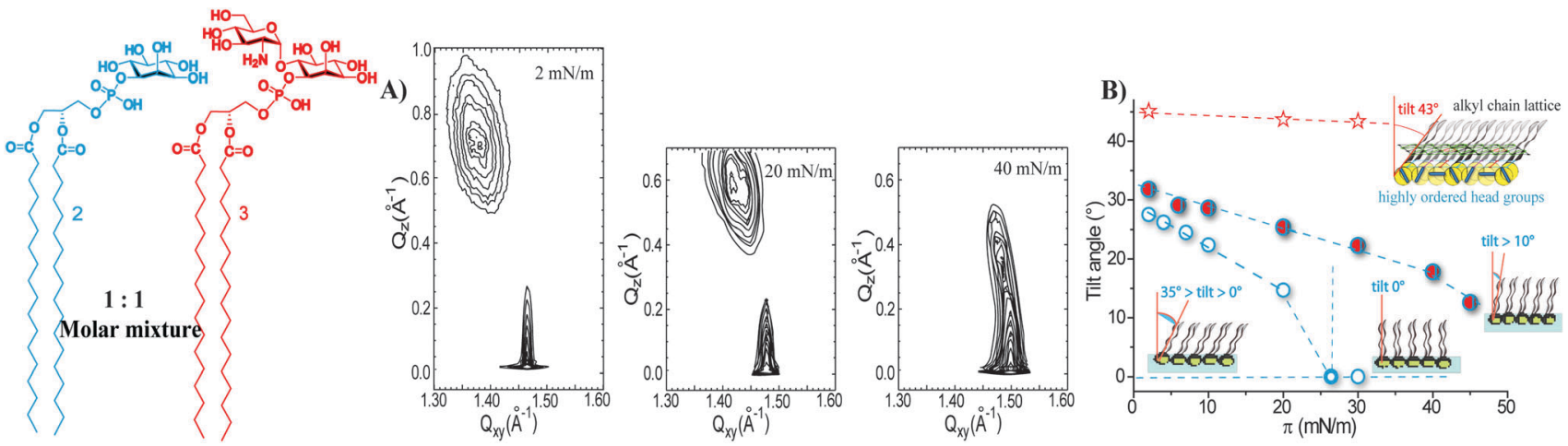

Fig. 7 (A) GIXD contour plots of the two-component monolayers $2 / 3$ (molar ratio $1: 1$ ) measured on PBS at $20{ }^{\circ} \mathrm{C}$ at different lateral surface pressures (indicated); (B) comparative representation of monolayer structural changes of the pure GPI-fragments ( $\mathbf{2}$ - light blue circles, $\mathbf{3}$ - red empty stars) and of their binary 1:1 mixture (2/3 - red filed blue circles) depicted by the variation of the tilt angle of the alkyl chains $(t)$ with the lateral surface pressure $(\pi)$.

in-plane packing of molecules and even the formation of a nontilted phase in mixed monolayers. The $\mathbf{1} / \mathbf{3}$ binary assembly behaves as a homogeneous entity with structural parameters (an area needed at the interface, a tilt angle) closer to those of the pure monolayer of $\mathbf{1}$ (Fig. 6C).

Mixed monolayers of the GPI-fragments 2 and 3 have been prepared by co-spreading the $1: 1$ (molar ratio) mixtures at the air/water interface. Similar to the mixed $\mathbf{1 / 3}$ monolayers, the mixed 2/3 monolayers also form a homogeneous structure (Fig. 7A), characterized only by the ordering of the alkyl chains. The Bragg peaks defining the supercell (low values of $Q_{x y}$ ) in the pure monolayers of 3 are absent indicating that 2 and 3 are completely miscible at 1:1 molar ratio. Van der Waals interactions of the alkyl chains control the monolayer packing and prevent the formation of a head group network encountered in monolayers of pure component 3 (Fig. $4 \mathrm{~A}$ and B).

Compared to the $1 / 3$ mixed system, the mixed $2 / 3$ monolayers are characterized by larger tilt angles of the ordered alkyl chains (Fig. 7B) owing to the increase in the steric demand of the head group going from 1 to 2 . The maximum tilt angle of the alkyl chains at zero surface pressure amounts to $31^{\circ}$ and the chains become non-tilted with respect to the surface normal only above $54 \mathrm{mN} \mathrm{m}^{-1}$ (Fig. S2, ESI $\dagger$ ).

\section{Conclusions}

A comparative analysis of the structural arrangement in a series of 2D model membranes of three GPI-fragments (monolayers formed at the air/water interface) demonstrates that increase in the size of the head groups from 1 to 2 and then 3 results in respective increase in the in-plane area per molecule that causes increase in the tilt of the alkyl chains and increase in surface pressure required for the transition to a non-tilted phase. While the trends observed for changes in tilt angles and transition surface pressure are in line with what is expected for such a series of glycophospholipids, the addition of a glucosamine moiety in compound 3 causes dramatic changes in the structure of the single component monolayers.

Compounds $\mathbf{1}$ and $\mathbf{2}$ form ordered monolayers defined only by an alkyl chain lattice. In contrast, GPI-fragment 3 forms higher ordered monolayers characterized by two commensurate lattices: a lattice of the alkyl chains and a molecular lattice formed as a consequence of ordering of the head groups through hydrogen bond interactions between glycans. These interactions are likely responsible for the partial segregation of GPI-fragment 3 when mixed with a liquid-disordered model membrane phospholipid (POPC). When compound 3 is mixed with lipids that form ordered monolayer phases, such as $\mathbf{1}$ or $\mathbf{2}$, hydrophobic interactions of the chains induce complete mixing of the two components. These interactions overcome the head group interactions responsible for ordering of entire molecules in the pure monolayers of compound 3. The mixed monolayers of $\mathbf{1}$ or $\mathbf{2}$ with $\mathbf{3}$ are homogenous with structures defined only by ordered alkyl chains and characterized by packing parameters that are dominated by the compounds not exhibiting strong head group interactions. To understand if this behaviour is generally applicable to more representative components of the cellular membranes, the ongoing studies are focused on the miscibility behaviour of GPI-fragment 3 with model membrane phospholipids able to form ordered phases. These studies, together with the details of the behaviour of unsaturated GPIs in model membranes, ${ }^{29}$ may further the understanding of the roles that lipid remodelling plays in the biosynthesis and intracellular transport of GPI-APs. ${ }^{13}$

\section{Acknowledgements}

This work was supported by the Max Planck Society and RIKEN-Max Planck Joint Center for Systems Chemical Biology. The research leading to these results has received funding from the European Commission's Seventh Framework Programme FP7/2007-2013 (postdoctoral fellowship to I.V.) and from the DFG (BR 1378/11-1). We are grateful to Mr Sebastian Götze for providing a building block for the synthesis of 3. We thank HASYLAB at DESY, Hamburg, Germany, for beamtime and excellent support.

\section{Notes and references}

1 Y.-H. Tsai, M. Grube, P. H. Seeberger and D. Varon Silva, Trends Glycosci. Glycotechnol., 2012, 24, 231-243. 
2 M. G. Paulick and C. R. Bertozzi, Biochemistry, 2008, 47, 6991-7000.

3 M. A. Ferguson, J. Cell Sci., 1999, 112, 2799-2809.

4 S. Schuck and K. Simons, J. Cell Biol., 2006, 172, 963-965.

5 S. Mayor and H. Riezman, Nat. Rev. Mol. Cell Biol., 2004, 5, 110-120.

6 M. Fujita and T. Kinoshita, Biochim. Biophys. Acta, 2012, 1821, 1050-1058.

7 K. Simons and D. Toomre, Nat. Rev. Mol. Cell Biol., 2000, 1, 31-39.

8 D. Kooyman, G. Byrne, S. McClellan, D. Nielsen, M. Tone, H. Waldmann, T. Coffman, K. McCurry, J. Platt and J. Logan, Science, 1995, 269, 89-92.

9 K. Simons and E. Ikonen, Nature, 1997, 387, 569-572.

10 I. Levental, M. Grzybek and K. Simons, Biochemistry, 2010, 49, 6305-6316.

11 C. Eggeling, C. Ringemann, R. Medda, G. Schwarzmann, K. Sandhoff, S. Polyakova, V. N. Belov, B. Hein, C. von Middendorff, A. Schonle and S. W. Hell, Nature, 2009, 457, 1159-1162.

12 Y. Wang, Y. Murakami, T. Yasui, S. Wakana, H. Kikutani, T. Kinoshita and Y. Maeda, J. Biol. Chem., 2013, 288, 25490-25499.

13 M. Fujita and Y. Jigami, Biochim. Biophys. Acta, 2008, 1780, 410-420.

14 Y.-H. Tsai, S. Goetze, I. Vilotijevic, M. Grube, D. V. Silva and P. H. Seeberger, Chem. Sci., 2013, 4, 468-481.

15 I. Vilotijevic, S. Götze, P. H. Seeberger and D. V. Silva, Modern Synthetic Methods in Carbohydrate Chemistry, Wiley-VCH, 2013, pp. 335-372.
16 C. Stefaniu, I. Vilotijevic, M. Santer, D. V. Silva, G. Brezesinski and P. H. Seeberger, Angew. Chem., Int. Ed., 2012, 51, 12874-12878.

17 G. Brezesinski, A. Dietrich, B. Struth, C. Böhm, W. G. Bouwman, K. Kjaer and H. Möhwald, Chem. Phys. Lipids, 1995, 76, 145-157.

18 C. Böhm, H. Möhwald, L. Leiserowitz, J. Als-Nielsen and K. Kjaer, Biophys. J., 1993, 64, 553-559.

19 K. Kjaer, Physica B, 1994, 198, 100.

20 D. Vollhardt, G. Brezesinski, S. Siegel and G. Emrich, J. Phys. Chem. B, 2001, 105, 12061-12067.

21 J. G. Petrov, G. Brezesinski, N. Krasteva and H. Möhwald, Langmuir, 2001, 17, 4581-4592.

22 M. N. Antipina, I. Schulze, B. Dobner, A. Langner and G. Brezesinski, Langmuir, 2007, 23, 3919-3926.

23 W. G. Ellenbroek, Y.-H. Wang, D. A. Christian, D. E. Discher, P. A. Janmey and A. J. Liu, Biophys. J., 2011, 101, 2178-2184.

24 M. Wehle, I. Vilotijevic, R. Lipowsky, P. H. Seeberger, S. D. Varon and M. Santer, J. Am. Chem. Soc., 2012, 134, 18964-18972.

25 E. Scalas, G. Brezesinski, V. M. Kaganer and H. Möhwald, Phys. Rev. E: Stat. Phys., Plasmas, Fluids, Relat. Interdiscip. Top., 1998, 58, 2172-2178.

26 J. Israelachvili, Colloids Surf., A, 1994, 91, 1-8.

27 D. Marsh, Handbook of Lipid Bilayers, CRC, Boca Raton, 1990.

28 R. F. M. de Almeida, A. Fedorov and M. Prieto, Biophys. J., 2003, 85, 2406-2416.

29 G. van Meer, D. R. Voelker and G. W. Feigenson, Nat. Rev. Mol. Cell Biol., 2008, 9, 112-124. 